\title{
Uma contribuição do design emocional para o desenvolvimento de produtos com abordagem do slow fashion
}

\author{
A contribution of emotional design to the development of products with a slow \\ fashion approach
}

SAPPER, Stella Lisboa; Mestra; Universidade Federal do Rio Grande do Sul

stellasapper@gmail.com

PIZZATO, Gabriela Zubaran de Azevedo; Doutora; Universidade Federal do Rio Grande do Sul

gabrielapizzato@gmail.com

JACQUES, Jocelise Jacques de; Doutora; Universidade Federal do Rio Grande do Sul

jocelisej@gmail.com

TEIXEIRA, Fábio Gonçalves; Doutor; Universidade Federal do Rio Grande do Sul

fabiogt@ufrgs.br

\section{Resumo}

Emoções podem auxiliar no relacionamento duradouro entre usuário e produto. A interação do usuário com experiências e objetos emocionalmente duráveis, pode diminuir o descarte e incentivar práticas de consumo mais conscientes, como as situadas na cultura slow. Diante de uma cultura de moda que prevalece o modelo fast fashion, afirma-se a necessidade de explorar práticas mais responsáveis. O objetivo deste trabalho é identificar como o design emocional pode contribuir para o desenvolvimento de produtos com abordagem do slow fashion, com foco na sustentabilidade. Por meio de uma revisão de literatura, foram apresentados conceitos que fundamentam as discussões em torno das contribuições do design emocional para o slow fashion. Foi identificada a importância e a influência que as emoções têm no desenvolvimento de produtos. Como resultado, os designers podem explorar esse campo, utilizando os conceitos do design emocional a fim de contribuir no projeto de produtos voltados para o slow fashion.

Palavras Chave: design de moda; design emocional; slow movement e moda sustentável.

\begin{abstract}
Emotions can help in the long-term relationship between user and product. By providing user interaction with emotionally durable experiences and objects, it is possible to decrease discarding and encourage more conscious consumer practices, such as those in the slow culture. Faced with a fashion culture that prevails the model of fast fashion market, it is affirmed the need to encourage more responsible practices. The objective of this work is to identify how emotional design can contribute to the development of products with a slow fashion approach. We presented concepts that underlie the discussions about the contributions of the emotional design to the slow fashion. As a result, designers can explore this field using these concepts.
\end{abstract}

Keywords: fashion design; emotional design; slow movement and sustainable fashion. 


\section{Introdução}

A moda sempre impôs um ciclo de vida para seus produtos, prevalecendo o sistema sazonal (primavera/verão e outono/inverno). De acordo com Cobra (2007, p.26), esse ciclo tende a ser cada dia mais curto, o que significa que a moda é mais evolucionária do que revolucionária. $\mathrm{A}$ moda se transforma ao longo do tempo e essas transformações requerem uma velocidade que deve estar relacionada não só à criação de novos produtos e à produção, mas também a um conjunto de estratégias que favoreçam o consumo rápido e uma resposta ágil do consumidor.

Na década de 1990, surgiu na Europa o modelo de mercado conhecido como fast fashion, termo que a imprensa especializada atribuiu ao fenômeno da moda rápida que estava ocorrendo em algumas lojas varejistas (CIETTA, 2010). Segundo Erner (2005), o fast fashion é baseado no Sentier, bairro parisiense conhecido por ser especializado na atividade têxtil e é considerado o inventor de uma maneira original de produzir roupas, utilizando um circuito curto, anglicizado como Quick Response System (sistema de resposta rápida) (ERNER, 2005, p. 146). Com isso, o fast fashion seria a forma industrial de aplicar o circuito curto, criado pelos comerciantes do Sentier.

O fast fashion é um modelo de mercado composto por várias estratégias de produção e marketing. O modelo não se baseia somente em uma velocidade rápida de produção e abastecimento, mas atua com uma ampla visão de mercado. A consolidação do fast fashion também ocorreu pela eficácia das empresas em resolver problemas-chaves do processo produtivo da moda, como o risco da imprevisibilidade da procura, a gestão do sistema criativo e da cadeia produtiva, e a definição de estratégias com foco no feedback do consumidor (CIETTA, 2010).

Com o crescimento do fast fashion diversas marcas se adaptaram, diminuindo o tempo de criação para atender a demanda da procura de novos produtos. A expansão desse modelo de mercado não se relaciona somente com o crescimento das empresas pioneiras, como Zara e H\&M, mas também ao aparecimento de consumidores das classes A e B em lojas antes consideradas populares, e a todas as estratégias do fast fashion adotadas por empresas convencionais, que empregavam o ciclo sazonal da moda. Esse comportamento de mercado é preocupante, visto que se espera que haja uma mudança nas prioridades de empresas que hoje adotam o fast fashion, e não que estas sejam um modelo a ser seguido. As estratégias aplicadas pelas empresas incentivam o consumo impulsionado pelo desejo, ocasionando uma proliferação de produtos no mercado e o descarte de um grande número de peças. Muitas empresas possuem condições de trabalho precárias e adotam um ciclo de vida bastante nocivo ao meio ambiente (CIETTA, 2010).

Nesse contexto de crescimento do fast fashion, coleções que eram lançadas semestralmente, passaram a ser lançadas mensalmente, semanalmente, chegando ao "see now, buy now" ("veja agora, compre agora"), onde as peças da coleção são disponibilizadas para compra imediatamente após o desfile (CIETTA; ROSSI, 2016). O conceito "see now, buy now" foi utilizado pela primeira vez em 2016 nas semanas de moda de Nova lorque, e já foi aplicado por marcas brasileiras, como Riachuelo e Ellus 2nd Floor.

Diante do sistema de velocidade rápida, existem outras visões que reconhecem não apenas a velocidade econômica, mas também a velocidade do meio ambiente e o ritmo de mudança cultural. Ideias de design, produção e consumo lento começaram a ser desenvolvidas no Slow Food Movement, fundado por Carlo Petrini na Itália, em 1986. Esse movimento foi o impulsionador de diversos movimentos de desaceleração, como o Slow Cities e o Slow Fashion (FLETCHER, 2011). A abordagem lenta incentiva a garantia de qualidade (do ambiente, da 
sociedade, da remuneração, das condições de trabalho, dos produtos, etc.), ética, criatividade e conexão com o meio ambiente (CARVALHAL, 2016; FLETCHER, 2011).

$\mathrm{Na}$ sociedade contemporânea, as pessoas consomem muito mais valores imateriais do que materiais, predominando o sentido dos signos e simulacros presentes nos produtos. A valorização do imaterial ocasionou um desprendimento do indivíduo com as coisas materiais, facilitando o descarte e a substituição (BAUDRILLARD, 1995; LIPOVETSKY, 2004). Na lógica do consumo, a emoção pode ser um fator determinante para evitar o descarte e estimular uma visão de mundo diferente. Para isso, não basta provocar uma resposta emocional no usuário em uma única ocasião, é necessário desenvolver uma relação entre usuário e objeto por um período prolongado de tempo. Por meio da emoção, é possível direcionar os usuários para além do mundo efêmero, a favor da interação com objetos emocionalmente duráveis e experiências positivas (CHAPMAN, 2005).

Para o design a emoção pode ser um fator fundamental no ato de projetar. Nesse contexto, conceitos do design emocional podem elucidar práticas para abordar as emoções nos projetos de design. De acordo com Norman (2004) e Walter (2011), o design emocional diz respeito as experiências do usuário com o produto e os vínculos estabelecidos pelas emoções provenientes dessas experiências. Por meio do design emocional, é possível tornar os usuários engajados com os produtos, transmitindo suas experiências positivas a outros potenciais usuários. Além disso, é possível estabelecer segurança e confiança aos usuários, incentivando a fidelização de marcas e redução do consumo exacerbado (NORMAN, 2004; WALTER, 2011).

Dada a importância das emoções para o consumo, o objetivo deste estudo é identificar como o design emocional pode contribuir para o desenvolvimento de produtos com abordagem do slow fashion, com foco em processos sustentáveis. Foi realizada uma revisão de literatura, onde foi constatada a necessidade dessa discussão. Este trabalho traz considerações teóricas que justificam a necessidade de estudos que tratam da relação entre o design emocional e o slow fashion.

\subsection{Procedimentos metodológicos}

A primeira etapa deste estudo envolveu uma revisão bibliográfica sistemática (RBS) com os procedimentos indicados por Conforto, Amaral e Silva (2011). Foram pesquisados artigos publicados internacionalmente entre os anos de 2011 e 2017 na bases de dados Periódicos CAPES, Web of Science e Science Direct. Após o teste de diferentes termos de busca, foram utilizadas as seguintes strings: "slow fashion" AND "emotion"; "slow fashion" AND "emotional design"; "sustainable fashion" AND "emotion"; "sustainable fashion" AND "emotional design".

O somatório dos resultados das buscas totalizou vinte e três artigos. A partir da leitura preliminar do título, resumo e palavras-chave, os artigos foram selecionados por meio de critérios de inclusão. Os critérios utilizados foram: (i) artigos nos idiomas português ou inglês; e (ii) artigos que abordavam a relação entre os conceitos do design emocional e do slow fashion. Com a aplicação dos critérios de inclusão, foram selecionados quatro artigos, que estão explicitados no tópico "resultados e discussão".

Com a RBS foi possível observar o quão recentes e escassos são os estudos que relacionam o design emocional e o slow fashion. Nesse contexto, na segunda etapa do estudo foi realizada uma revisão de literatura a respeito do slow fashion e do design emocional para fundamentar a 
discussão e alcançar o objetivo. Os artigos selecionados na RBS norteiam a discussão das relações entre os dois campos.

\section{Slow Fashion}

O slow fashion objetiva reformular o uso da velocidade como uma força para a sustentabilidade, e não apenas como um promotor da descontinuidade, do consumo e da riqueza. Ao combinar as ideias do slow movement com os aspectos da indústria do vestuário, busca-se construir uma nova visão para a moda nos tempos da sustentabilidade. Nesse contexto, o prazer e a moda estão ligados à responsabilidade e à consciência. Esse movimento reconhece a importância da moda para a cultura de massa e a urgência da agenda de sustentabilidade. O slow fashion é sobre uma nova visão de mundo, sobre projetar, desenvolver, consumir e viver melhor (FLETCHER, 2011).

Apesar do termo sugerir, o slow fashion não é uma oposição ao fast fashion. Enquanto o fast fashion é um modelo de mercado bem definido, com estratégias de negócios e marketing, o slow fashion é uma abordagem diferente, onde designers, compradores, varejistas e consumidores têm mais consciência dos impactos dos produtos sobre os trabalhadores, comunidades e ecossistemas. Por meio dessa abordagem, as ideias são combinadas envolvendo aspectos do tempo da natureza, ciclos de regeneração e evolução, do tempo da cultura, do valor da tradição, e dos prazos aplicados na moda e no comércio. Frequentemente, pessoas que adotam uma visão de mundo slow acabam extrapolando para diversas áreas, como alguém que adota uma alimentação vegana e orgânica e começa a se preocupar com a origem do seu vestuário e dos demais produtos que consome (FLETCHER, 2011; CARVALHAL, 2016).

A cultura slow funciona como um convite para pensar em novos sistemas no setor da moda e questionar o papel do crescimento econômico e demais valores para que uma sociedade diferente se desenvolva. Reconhece-se que para mudar a moda, é necessário incluir as práticas econômicas e sociais no debate para a mudança (FLETCHER, 2010).

As abordagens com uma velocidade mais lenta produzem um número menor de produtos de alta qualidade. Assim, os preços dos itens com uma abordagem slow fashion, geralmente, são mais elevados do que os produtos de fast fashion. A alta qualidade e as estratégias de preços do slow fashion estimulam a percepção dos consumidores sobre o real valor do que estão comprando, encorajando-os a manter o item por mais tempo, evitando o descarte pós-compra (FLETCHER, 2011; JUNG; JIN, 2016).

Jung e Jin (2014) identificaram cinco subdimensões do slow fashion. Por meio de uma pesquisa com consumidores, foi identificado que o conceito do slow fashion envolve: equidade, autenticidade, funcionalidade, localismo e exclusividade. A dimensão "equidade" indica que os consumidores estão atentos a práticas de trabalho quando compram roupas, como comércio, compensação e um ambiente de trabalho justo para os produtores. A dimensão "autenticidade" inclui a apreciação dos consumidores por roupas feitas com métodos tradicionais e artesanais de construção. A dimensão "funcionalidade" inclui as preocupações dos consumidores com a versatilidade e longevidade do vestuário. A dimensão "localismo" diz respeito ao apoio as marcas nacionais e a importância percebida no uso de matérias-primas locais. Não se limitando às comunidades locais, o "localismo" envolve a preferência por marcas nacionais em detrimento das marcas globais de vestuário. A dimensão "exclusividade" se refere a valorização dos consumidores por roupas exclusivas por meio da compra de edições limitadas ou itens raros (JUNG; JIN, 2014) 
Com uma abordagem voltada ao humano, o movimento slow garante os direitos humanos fundamentais aos trabalhadores, eliminando a pressão do tempo de produção na cadeia do vestuário e garantindo uma remuneração justa. Cons equentemente, a qualidade é elevada devido ao tempo gasto em cada peça. No sistema de produção de velocidade mais baixa, os designers podem trabalhar em conjunto com os consumidores, para promover uma aproximação que auxilie no processo de criação de forma a atender as necessidades dos consumidores. Com uma ligação entre projetistas e consumidores, tais processos de co-criação incentivam um comportamento mais responsável, devido a uma conscientização sobre como uma peça de vestuário é feita (CATALDI; DICKSON; GROVER, 2010).

Em outra pesquisa com consumidores, realizada por Pookulangara e Shephard (2013), foi possível observar o quanto a informação a respeito do processo de produção influencia na decisão de compra, porém, a transparência continua sendo um desafio para muitas empresas, além de muitas das informações não estarem ao alcance dos consumidores. A comunicação aberta com os consumidores é outra característica do slow fashion, e é fundamental para a conscientização e melhores práticas. Alguns consumidores também indicam a dificuldade em adquirir peças devido ao valor acima da média do mercado (POOKULANGARA; SHEPHARD, 2013). Apesar do alto custo de algumas peças com abordagem slow fashion, consequência do processo produtivo, o movimento slow também se caracteriza pelo incentivo de práticas alinhadas com ações sustentáveis, como reciclagem, upcycling, aumento do ciclo de vida e práticas zero waste (desperdício zero) (FLETCHER, 2011).

Moda e vestuário contribuem para o bem-estar humano, tanto no aspecto funcional quanto emocional. Fletcher (2011) indica que enquanto a moda conecta o indivíduo no tempo e espaço e lida com as necessidades emocionais, o vestuário atende as necessidades físicas e funcionais, como proteção e conforto térmico. O vestuário é uma produção material e a moda é uma produção simbólica, que pode ou não estar manifestada em um bem material. No entanto, onde a moda e o vestuário se unem, por meio das roupas de moda, as necessidades emocionais alimentam o consumo de recursos, gerando desperdício e um sistema de descontinuidade através das tendências sazonais. Com isso, o indivíduo se torna insatisfeito, pois os bens materiais nunca irão satisfazer verdadeiramente as necessidades psicológicas.

Nesse contexto, é necessário reconhecer essas diferenças entre moda e vestuário e desenvolver projetos mais conscientes. Deve-se reconhecer a moda como parte significativa da cultura e separá-la do consumo desenfreado, produzindo roupas baseadas em valores, com materiais cuidadosamente pensados, com um processo sustentável e que tenham apelo estético e simbólico (FLETCHER, 2011). Dessa forma, o designer por meio de novas práticas e métodos pode auxiliar no desenvolvimento de objetos e experiências emocionalmente duráveis, diminuindo o ciclo de descarte e incentivando práticas mais conscientes de consumo.

\section{Design Emocional}

No cenário internacional ao se falar em "design emocional", entende-se como o emprego de teorias provenientes de relações entre as áreas da psicologia, do design e da pesquisa. Nesse contexto, a emoção pode ser previsível e controlável, e o processo de design pode moldar experiências emocionais desejadas. Para isso, a área busca entender como projetar para evocar ou evitar certas emoções, com foco na aproximação com o usuário e no entendimento de suas necessidades (TONETTO; COSTA, 2011). 
Desmet e Hekkert (2009) identificaram quatro abordagens utilizadas pelos autores para integrar a emoção do usuário no processo de design: com foco no usuário, com foco no designer, com foco na pesquisa e com foco em teoria. A abordagem com foco no usuário busca envolver os usuários no processo, usando suas aspirações e sentimentos como força criativa. São utilizados técnicas e métodos de co-criação e técnicas generativas, como colagens e maquetes. Essa abordagem se baseia na ideia que o usuário pode ser envolvido tanto nos estágios iniciais do projeto quanto em testes mais avançados. Na abordagem com foco no designer, o projetista desempenha um papel mais autônomo. Os designers são vistos como autores que comunicam ideias, que buscam desafiar os usuários com novos produtos ao invés de agradá-los. Nessa abordagem, o design emocional pode ser interpretado como uma manifestação dos princípios ou visões do designer. Em abordagens com foco na pesquisa, são utilizados métodos e técnicas para mensuração das emoções, que auxiliam nas decisões de projeto. Pode-se citar o trabalho de Desmet (2002) que apresenta um instrumento completo para medir um conjunto de 18 emoções positivas e negativas, e a Engenharia Kansei (NAGAMACHI, 1995). As abordagens com foco em teoria ampliam as pesquisas com perspectivas teóricas avançadas sobre como produtos provocam emoções. Isso pode gerar insights a respeito da influência do produto sobre as emoções e podem auxiliar na geração de novas relações entre produto e usuário (DESMET; HEKKERT, 2009).

O design emocional também pode ser explicado por meio dos três níveis de processamento propostos por Norman (2004): visceral, comportamental e reflexivo. Cada um dos níveis desempenha papel importante na experiência e requer uma abordagem de design diferente.

O nível visceral envolve o design para aparência e o que a natureza faz. Os sinais emocionais recebidos do ambiente são interpretados automaticamente no nível visceral. Nesse nível, os consumidores recebem sinais pela primeira impressão, resultante da experiência e, em seguida, esboçam suas reações iniciais. Quando algo é percebido como "atrativo", es se julgamento vem diretamente do nível visceral. Os princípios subjacentes ao design visceral são interligados, consistentes entre culturas e pessoas, com predominância das características físicas. A sensação física e a textura dos materiais são importantes no nível visceral, assim como o impacto emocional imediato (NORMAN, 2004).

O nível comportamental envolve o design para o prazer e eficácia do uso. Nesse nível a aparência e a fundamentação não são importantes, o que importa é o desempenho. Para uma boa concepção comportamental deve-se atender aos seguintes componentes: função, compreensão, usabilidade e sensação física. Nesse contexto, a função está em primeiro lugar. Em um projeto de design comportamental deve-se compreender as necessidades do usuário, por meio de estudos de comportamento em diferentes ambientes. Em seguida, a equipe de projeto deve produzir protótipos rápidos e teste com usuários. Esse processo é iterativo e centrado no usuário, até que se chegue a uma solução de design satisfatória (NORMAN, 2004).

O nível reflexivo envolve o design reflexivo. Esse nível envolve tudo sobre a mensagem, a cultura e o significado de um produto ou de seu uso. Embora a atratividade (resposta à aparência superficial do objeto) seja um fenômeno de nível visceral, a percepção da beleza vem do nível reflexivo. A beleza aqui vem da reflexão consciente e da experiência do usuário e é influenciada pela cultura, conhecimento e aprendizagem. Mesmo objetos que não são atraentes superficialmente podem propiciar prazer no uso. Projetar em nível reflexivo pressupõe a compreensão de significados para as pessoas (NORMAN, 2004). 
Ao abordar emoção no ciclo de design, conforme observado anteriormente, é necessário abordar três componentes principais: designers, o resultado do processo de design e os usuários (HO; SIU, 2012). Com foco nas relações entre esses três componentes, Ho e Siu (2012) propuseram em seu trabalho uma estrutura, intitulada modelo $3 E$, que apresenta as relações entre os conceitos emotion design, emotional design, emotionalise design, e os três níveis de processamento de Norman (2004). Os três componentes - designers, design (resultado) e usuários - estão sempre incluídos no processo de design e criam uma estrutura importante.

Com base em uma revisão de literatura, Ho e Siu (2012) definem os conceitos a seguir. Conforme já visto, o emotional design diz respeito a emoção provocada pelo produto no usuário e envolve os três níveis de processamento de informação - visceral, comportamental e reflexivo. 0 emotionalise design envolve a influência das emoções dos designers nos processos de design e nos resultados alcançados. Os resultados de design que incluem aspectos emocionais, utilizando métodos intuitivos e emocionais, podem auxiliar no estabelecimento de uma relação mais próxima entre designers e usuários. Emotion design é um conceito mais amplo, que envolve a interação entre designers e usuários em função de um projeto ou produto. $O$ design contribui com a experiência e comunica ideias (HO; SIU, 2012).

A Figura 1 apresenta o modelo $3 E$ e a dinâmica de interação dos componentes essenciais no ciclo de design com abordagem das emoções. Conforme pode ser observado, o fluxo de emoção ocorre tanto dos designers (equipe de projeto) para o design (produto), como do produto para os usuários/consumidores. Durante o processo de design, os designers acabam gerando um fluxo de emoção ao projetar os componentes do produto. Esses componentes, como aparência, forma e estilo, geram um fluxo de emoção perante os usuários, fazendo com que os mesmos consumam e tenham uma experiência com o produto. Por meio da compreensão dessas relações entre designer, produto e usuário é possível observar que a emoção vai além da relação usuárioproduto, e sim faz parte de todas as etapas do ciclo de design.

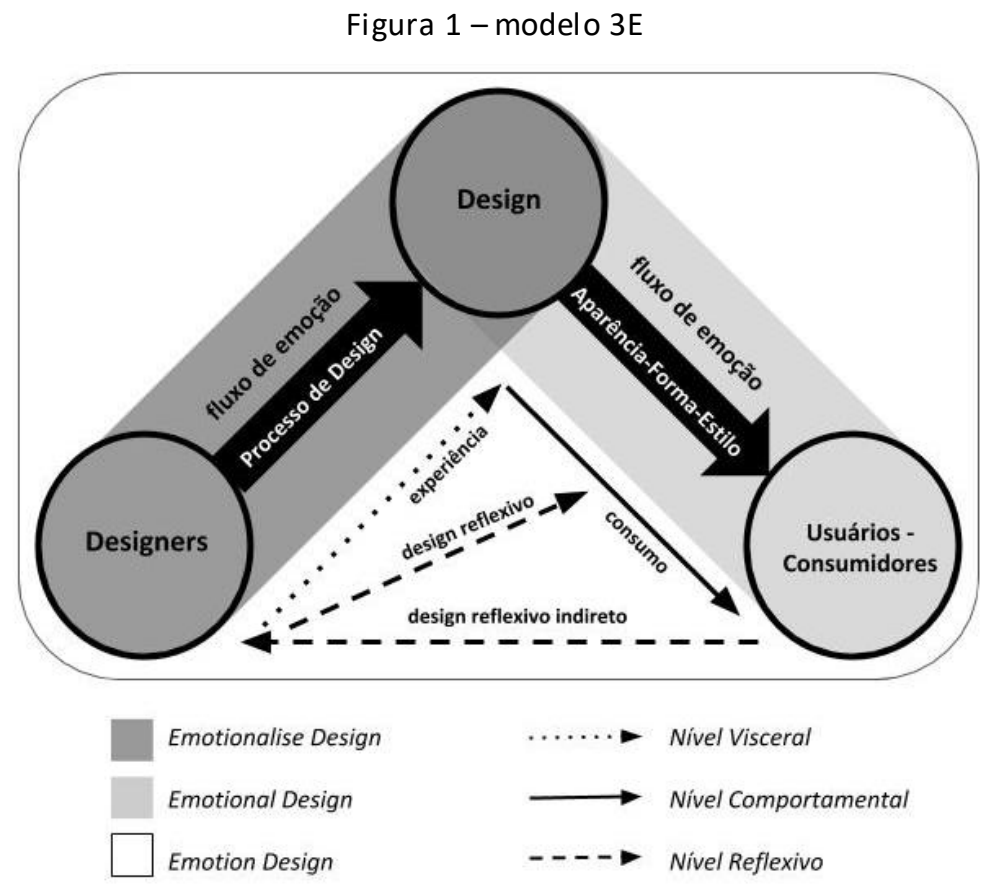

Fonte: traduzido de Ho e Siu (2012) 


\section{Resultados e discussão}

Neste tópico serão abordados os artigos selecionados na RBS, realizada na primeira etapa da pesquisa. Esses estudos abordam conceitos do slow fashion e do design emocional de forma a gerar novas estratégias para o desenvolvimento de produtos. A discussão também é delineada com outros estudos que fortalecem as ideias apresentadas nos artigos selecionados.

Por meio dos critérios de inclusão da RBS, explicitados no tópico "procedimentos metodológicos", foram selecionados quatro artigos (NIINIMÄKI; HASSI, 2011; LAPOLLA; SANDERS, 2015; PARK; KIM, 2016; POLDNER; DENTONI; IVANOVA, 2017). Os artigos excluídos não atendiam aos critérios de inclusão, eram repetidos ou abordavam outras áreas do conhecimento. A Tabela 1 apresenta os dados dos artigos selecionados.

Tabela 1 - dados dos artigos selecionados na RBS

\begin{tabular}{|c|c|c|c|c|}
\hline Periódico & Título & Autores & Ano & Palavras-chave \\
\hline $\begin{array}{l}\text { Clothing and } \\
\text { Textiles Research } \\
\text { Journal }\end{array}$ & $\begin{array}{l}\text { Using Cocreation to Engage } \\
\text { Everyday Creativity in Reusing and } \\
\text { Repairing Apparel }\end{array}$ & $\begin{array}{l}\text { LAPOLLA, K.; } \\
\text { SANDERS, E. B. } \\
\text { N. }\end{array}$ & 2015 & $\begin{array}{l}\text { creativity, cocreation, } \\
\text { reuse, repair, used apparel }\end{array}$ \\
\hline $\begin{array}{l}\text { Journal of } \\
\text { Cleaner } \\
\text { Production }\end{array}$ & $\begin{array}{l}\text { Emerging design strategies in } \\
\text { sustainable production and } \\
\text { consumption of textiles and } \\
\text { clothing }\end{array}$ & $\begin{array}{l}\text { NIINIMÄKI, K.; } \\
\text { HASSI, L. }\end{array}$ & 2011 & $\begin{array}{l}\text { textile industry, } \\
\text { sustainable design, } \\
\text { strategies, value creation, } \\
\text { strategic innovation }\end{array}$ \\
\hline $\begin{array}{l}\text { Journal of } \\
\text { Retailing and } \\
\text { Consumer } \\
\text { Services }\end{array}$ & $\begin{array}{l}\text { Proactive versus reactive apparel } \\
\text { brands in sustainability: Influences } \\
\text { on brand loyalty }\end{array}$ & $\begin{array}{l}\text { PARK, H.; } \\
\text { KIM, Y.-K. }\end{array}$ & 2016 & $\begin{array}{l}\text { sustainability, } \\
\text { sustainable fashion, } \\
\text { fast fashion, brand loyalty, } \\
\text { brand trust, } \\
\text { brand affect }\end{array}$ \\
\hline $\begin{array}{l}\text { Journal of } \\
\text { Cleaner } \\
\text { Production }\end{array}$ & $\begin{array}{l}\text { Aesthetic mediation of creativity, } \\
\text { sustainability and the organization }\end{array}$ & $\begin{array}{l}\text { POLDNER, K.; } \\
\text { DENTONI, D.; } \\
\text { IVANOVA, O. }\end{array}$ & 2017 & $\begin{array}{l}\text { aesthetic mediation, } \\
\text { fashion, creativity, } \\
\text { sustainability, } \\
\text { aesthetic inquiry, } \\
\text { organization }\end{array}$ \\
\hline
\end{tabular}

Fonte: os autores

A aproximação dos usuários no processo de design e a aplicação de técnicas provenientes do design emocional pode ser um ponto chave para a obtenção de produtos emocionalmente duráveis no campo do slow fashion. Lapolla e Sanders (2015) realizaram uma pesquisa utilizando métodos de co-criação para explorar oportunidades existentes para encorajar a criatividade individual na reutilização e reparação de roupas que seriam descartadas. Foram realizadas oficinas com mulheres de 20 a 60 anos, que geraram mapas visuais e novos artefatos. Pôde ser observado que os métodos de co-criação aplicados em vestuário podem incentivar a criatividade e a capacidade de adaptação dos participantes. Mesmo as oficinas curtas inspiraram as participantes a explorar uma gama maior de atividades criativas para reorientar o vestuário sem uso (LAPOLLA; SANDERS, 2015). 
Niinimäki e Hassi (2011) apresentam um conjunto de estratégias de projeto e fabricação para a indústria têxtil e de confecção para reduzir o impacto da produção e do consumo de massa. As estratégias indicadas pelas autoras envolvem os seguintes aspectos: garantia de satisfação e ciclo de vida longo; apego ao produto e design emocionalmente satisfatório; customização; cocriação; open source design e design de serviços. Todas essas estratégias objetivam aumentar o ciclo de vida do produto através de processos que favoreçam a interação produto-usuário. Destaca-se as duas primeiras estratégias, garantia de satisfação e ciclo de vida longo e apego ao produto e design emocionalmente satisfatório.

Uma das maiores insatisfações dos consumidores é a baixa qualidade do vestuário. Nesse sentido, os fornecedores poderiam oferecer informações aos consumidores sobre a vida útil prevista do produto, pois é difícil avaliar esse aspecto na hora da compra. No slow fashion objetiva-se prolongar a vida útil de um produto e aprofundar a satisfação do usuário (NIINIMÄKI; HASSI, 2011).

Quanto ao apego e ao design emocionalmente satisfatório, existem diversas estratégias para projetar maiores experiências emocionais e que, consequentemente, resultam em apego e satisfação emocional no usuário. Pode-se utilizar materiais que envelhecem de forma favorável, que desenvolvam uma nova qualidade ao longo do tempo. A funcionalidade também pode ser trabalhada dessa forma, quando o desempenho e capacidade de um produto melhora ao longo do tempo. O reparo também é fundamental ao projetar produtos que possuem fácil reparação, atualização e manutenção, pois no contexto de um design emocionalmente durável, a reparação é um processo de aperfeiçoamento para a frente, e não para o estado anterior (CHAPMAN, 2016).

Park e Kim (2016) identificaram, por meio de uma pesquisa com 556 consumidores americanos, as diferenças na formação de lealdade de consumidores de marcas de moda sustentável e de marcas de fast fashion. Os resultados forneceram evidências de que os consumidores desenvolvem mais confiança em marca proativas (marcas de moda sustentável, em particular), porém, o valor percebido pelo consumidor de marcas de moda sustentável se relaciona mais à confiança do que ao afeto. Consumidores de moda mais conscientes possuem grande confiança em marcas sustentáveis, porém são menos propensos a expressar afeto ou excitação por uma marca sustentável. Com isso, é importante que as marcas com viés sustentável promovam suas ações de forma transparente e se aproximem de seus consumidores em diversas vertentes para aumentar o afeto e o vínculo emocional em conjunto com a confiança.

Embora a sustentabilidade seja um campo vasto de estudo, a bibliografia sobre sustentabilidade muitas vezes se concentra em estudos mais técnicos, como avaliações de ciclo de vida, gestão da cadeia produtiva e práticas mais limpas de produção. Entretanto, em meio a tantas preocupações técnicas, existe um processo de criação que envolve criatividade e construção de valores estéticos e simbólicos, para além da função prática.

Nesse contexto, Poldner, Dentoni e Ivanova (2017) realizaram um estudo de como a estética pode funcionar como uma ferramenta de mediação para traduzir a criatividade em mensagens sustentáveis. Foi utilizada como evidência empírica a marca brasileira Osklen. A Osklen aplica uma forma de cultura de sustentabilidade, que incorpora a cada coleção temas sociais e sustentáveis que sejam significativos para representar e valorizar a essência do Brasil. No caso Osklen, evidências mostraram que a estética media o processo entre criatividade e comunicação de sustentabilidade por meio de quatro etapas, que orientam o indivíduo em transformar fontes 
de inspiração em uma coleção tangível: (1) abertura para novos fenômenos inspiradores; (2) ter uma epifania sobre um fenômeno (natural, social ou humano); (3) conectar a essência do fenômeno a uma mensagem de sustentabilidade; e (4) traduzir a essência do fenômeno em um produto tangível.

Com base na pesquisa estética de três coleções da Osklen, Poldner, Dentoni e Ivanova (2017) identificaram três técnicas que são aplicadas na última etapa, "traduzir a essência do fenômeno em um produto tangível". As técnicas são "preservação", "transformação" e "inserindo novidade". "Preservação" diz respeito a preservar as qualidades estéticas do fenômeno inspirador (como formas originais, cores, materiais), na técnica "transformação" o designer modifica características do fenômeno por meio de analogias e utiliza diferentes recursos de representação, por fim, a técnica "inserindo novidade" envolve a inserção de novas qualidades estéticas ao produto (ver POLDNER; DENTONI; IVANOVA, 2017).

Rivera et al. (2015) analisaram como os elementos imateriais se relacionam com a sustentabilidade. Para o desenvolvimento de um produto ou serviço voltado para a sustentabilidade é necessária uma abordagem multidisciplinar, porém deve-se atentar para abordagens centradas no usuário e para métodos de avaliação da experiência e das emoções do usuário. É interessante pensar o design centrado no usuário por meio de um viés sustentável em relação a identificação das necessidades, emoções, comportamentos e valores. Rivera et al. (2015) indicam pontos-chave de elementos imateriais que devem ser levados em conta em projetos sustentáveis, como: apego, admiração, envolvimento, satisfação, bem-estar, realização (cultural e espiritual), prazer, felicidade, liberdade (escolha e ação), desejo e conforto.

Por meio dos estudos apresentados anteriormente, foi possível sintetizar os resultados encontrados em diretrizes que apoiem projetos com abordagem slow fashion de maneira que aumentem o apelo emocional dos produtos. As diretrizes foram divididas em quatro grupos: (i) ciclo de vida, (ii) criação, (iii) estética e (iv) posicionamento. A Tabela 2 apresenta a síntese das diretrizes conforme conceitos discutidos neste trabalho.

Tabela 2 - Síntese das diretrizes identificadas nas referências

\begin{tabular}{ll}
\hline Referências & Diretrizes \\
\hline Niinimäki e Hassi (2011); & Ciclo de vida \\
Chapman (2016) & Prolongar a o máximo a vida útil do produto. \\
& Explicitar e informar os consumidores sobre a vida útil do produto. \\
& Adotar estratégias para aumentar as experiências emocionais. \\
Usar materiais resistentes e de qualidade. & Projetar funcionalidades que seadaptem ao longo do tempo. \\
Possibilitar o reparo dos produtos.
\end{tabular}

Lapolla e Sanders (2015);

Parke Kim (2016)

\section{Criação}

Aproximar os usuários do processo de desenvolvimento de produtos. Co-criar com os usuários.

Encorajar a reutilização de materiais e produtos descartados.
Poldner, Dentoni e Ivanova (2017);

Rivera et al.(2015)

\section{Estética}

Inspirar-se em temáticas sustentáveis (sociais, a mbientais ou culturais). Inserir qualida des estéticas no produto.

Avaliar os produtos com usuários (aspectos materiais e imateriais) 
Ser proativo em causas sociais, a mbientais e culturais.

Ter um processo transparente.

Promover ações condizentes com valores sustentáveis.

Valorizar a cultura local.

Fonte: os autores

\section{Considerações finais}

Por meio da RBS, foram identificados quatro artigos que relacionam o design emocional e o slow fashion. A RBS realizada no trabalho encontrou menos trabalhos que o esperado na área do design emocional e do slow fashion, demonstrando que a relação entre esses temas é recente e pouco explorada. Mesmo trabalhos que abordam os conceitos do design emocional na moda são escassos. Os artigos selecionados na RBS salientam a relevância da abordagem das emoções em projetos de slow fashion e de moda sustentável, porém pouco exploram métodos e técnicas mais estruturados e sistemáticos para serem aplicados no design de moda.

Diante do estudo dos artigos da RBS (NIINIMÄKI; HASSI, 2011; LAPOLLA; SANDERS, 2015; PARK; KIM, 2016; POLDNER; DENTONI; IVANOVA, 2017) e de outros estudos relevantes (RIVERA, et al., 2015; CHAPMAN, 2016) foi possível sintetizar os conceitos encontrados em diretrizes que auxiliem projetos com abordagem slow fashion. Grande parte das diretrizes se referem a processos mais transparentes e a um estreitamento da relação entre empresa, e/ou equipe de projeto, e os usuários finais. Apesar dos consumidores demonstrarem grande confiança em marcas sustentáveis (PARK; KIM, 2016), é necessário aumentar processos que estabeleçam vínculos emocionais além da confiança. Para que isso ocorra, é fundamental a aproximação das empresas com os usuários.

Ao abordar conceitos do design emocional, a estrutura (modelo $3 E$ ) proposta por Ho e Siu (2012) pode servir como ponto de partida para pesquisas mais aprofundadas a respeito das práticas do design emocional e do desenvolvimento de produtos com abordagem slow fashion. A compreensão das relações e interações entre designer, design (resultado do processo) e usuário, conforme apresentado no modelo $3 E$ (HO; SIU, 2012), é fundamental em qualquer processo de design, e ainda pouco explorado na área da moda.

Os resultados observados com a pesquisa apontam a importância da compreensão do contexto do slow fashion. Compreender como ocorre a interação entre produto e usuário (emotional design e níveis visceral, comportamental e reflexivo), como os designers podem se apropriar de estados emocionais e experiências para gerar soluções que estabeleçam uma relação estreita com o usuário (emotionalise design) e analisar as respostas geradas nesse ciclo com o objetivo de gerar produtos emocionalmente duráveis e estratégias efetivas rumo à sustentabilidade.

Profissionais da área do design e da moda podem explorar e contribuir com projetos e estudos que relacionem o design emocional ao slow fashion. Ampliar os estudos nessa área pode resultar no surgimento de melhores práticas no contexto projetual, assim como difundir a temática na área do design. 


\section{Referências}

BAUDRILLARD, J. A sociedade de consumo. Lisboa: Ed. 70, 1995.

CARVALHAL, A. Moda com propósito: manifesto pela grande virada. São Paulo: Paralela, 2016.

CATALDI, C.; DICKSON, M.; GROVER, C. Slow fashion: tailoring a strategic approach towards sustainability. Master's thesis, Blekinge Institute of Technology, Sweden, 2010.

CHAPMAN, J. Design, emotion and longer lasting products - Deliver specific insights to designers how to create emotionally durable products. Proceedings 10th International Conference on Design \& Emotion, p. 74-79, 2016.

CHAPMAN, J. Emotionally durable design: objects, experiences and empathy London: Earthscan, 2005.

CIETTA, E. A revolução do fast fashion: estratégias e modelos organizativos para competir nas indústrias híbridas. São Paulo: Estação das Letras e Cores, 2010.

CIETTA, E.; ROSSI, P. "See now, buy now" runway shows and slow fashion: and supposing it suits "made in italy" to jump on bandwagon? Area Studi Diomedea, 2016.

COBRA, M. Marketing e moda. São Paulo: Cobra Editora e Marketing, 2007.

CONFORTO, E. C.; AMARAL, D. C.; SILVA, S. L. DA. Roteiro para revisão bibliográfica sistemática: aplicação no desenvolvimento de produtos e gerenciamento de projetos. In: $8^{\circ}$ Congresso Brasileiro de Gestão de Desenvolvimento de Produto - CBGDP 2011. Porto Alegre, RS, Brasil. Instituto de Gestão de Desenvolvimento do Produto - IGDP. Anais... Porto Alegre: IGDP, 2011.

DESMET, P. Designing Emotions. Delft: Delft University of Technology, 2002.

DESMET, P.; HEKKERT, P. Special Issue Editorial: Design \& Emotion. International Journal of Design, v. 3(2), p.1-6, 2009.

ERNER, G. Vítimas da moda? Como a criamos, por que a seguimos. Tradução Eric Roland René Heneault. São Paulo: Editora Senac São Paulo, 2005.

FLETCHER, K. Slow fashion: an invitation for systems change. Fashion Practice, v. 2, p. 259-266, 2010.

FLETCHER, K. Sustainable fashion and textiles design journeys. London: Earthscan, 2011.

JUNG, S.; JIN, B. From quantity to quality: understanding slow fashion consumers for sustainability and consumer education. International Journal of Consumer Studies, v. 40, p.410-421, 2016.

JUNG, S.; JIN, B. A theoretical investigation of slow fashion: sustainable future of the apparel industry. International Journal of Consumer Studies, v. 38, p.510-519, 2014.

HO, A. G.; SIU, K. Emotion design, emotional design, emotionalize design: a review on their relationships from a new perspective. The Design Journal, v. 15, p. 9-32, 2012.

LAPOLLA, K.; SANDERS, E. B.-N. Using co creation to engage everyday creativity in reusing and repairing apparel. Clothing and Textiles Research Journal, v. 33, p. 183-198, 2015.

LIPOVETSKY, G. Os tempos hipermodernos. São Paulo: Editora Barcarolla, 2004.

NAGAMACHI, M. Kansei engineering: a new ergonomic consumer-oriented technology for product 
development. International Journal of Industrial Ergonomics, v. 15, p.3-11, 1995.

NIINIMÄKI, K; HASSI, L. Emerging design strategies in sustainable production and consumption of textiles and clothing. Journal of Cleaner Production, v. 19, p.1876-1883, 2011.

NORMAN, D. Emotional design: why we love (or hate) everyday things. New York: Basic Books, 2004.

PARK, H.; KIM, Y.-K. Proactive versus reactive apparel brands in sustainability: influences on brand loyalty. Journal of Retailing and Consumer Services, v. 29, p.114-122, 2016.

POLDNER, K.; DENTONI, D.; IVANOVA, O. Aesthetic mediation of creativity, sustainability and the organization. Journal of Cleaner Production, v. 140, p.1936-1947, 2017.

POOKULANGARA, S.; SHEPHARD, A. Slow fashion movement: Understanding consumer perceptions -an exploratory study. Journal of Retailing and Consumer Services, v. 20, p.200-2006, 2013.

RIVERA, J. C. et al. Immaterial elements as drivers of sustainability in products and services. The 22nd CIRP conference on Life Cycle Engineering, p.615-620, 2015.

TONETTO, L.; COSTA, F. Design emocional: conceitos, abordagens e perspectivas de pesquisa. Strategic Design Research Journal, v. 4 (3), p.132-140, 2011.

WALTER, A. Designing for emotion. New York: A Book Apart, 2011. 